\title{
Sphenopalatine ganglion neuromodulation in migraine: What is the rationale?
} DOI: I0.1 I 77/0333 I024I35I 2032 cep.sagepub.com

$\ominus$ SAGE

\section{Sabrina Khan', Jean Schoenen ${ }^{2}$ and Messoud Ashina'}

\begin{abstract}
Objective: The objective of this article is to review the prospect of treating migraine with sphenopalatine ganglion (SPG) neurostimulation.

Background: Fuelled by preliminary studies showing a beneficial effect in cluster headache patients, the potential of treating migraine with neurostimulation has gained increasing interest within recent years, as current treatment strategies often fail to provide adequate relief from this debilitating headache.

Common migraine symptoms include lacrimation, nasal congestion, and conjunctival injection, all parasympathetic manifestations. In addition, studies have suggested that parasympathetic activity may also contribute to the pain of migraineurs.

The SPG is the largest extracranial parasympathetic ganglion of the head, innervating the meninges, lacrimal gland, nasal mucosa, and conjunctiva, all structures involved in migraine with cephalic autonomic symptoms.

Conclusion: We propose two possible mechanisms of action: I) interrupting the post-ganglionic parasympathetic outflow to inhibit the pain and cephalic autonomic symptoms, and 2) modulating the sensory processing in the trigeminal nucleus caudalis. To further explore SPG stimulation in migraineurs as regards therapeutic potential and mode of action, randomized clinical trials are warranted.
\end{abstract}

\section{Keywords}

Sphenopalatine ganglion, migraine, trigeminovascular pathway, trigemino-parasympathetic reflex, neurostimulation, neuromodulation

Date received: 30 July 20I3; revised: 6 October 20I3; accepted: 6 October 2013

\section{Introduction}

Migraine is a common neurological syndrome, rated as one of the most severe disabling disorders by the World Health Organization (1). It is managed pharmacologically with acute and preventive drug therapies. However, current treatment strategies are often suboptimal in terms of inadequate efficacy and substantial side effects (2). New treatment modalities are therefore warranted to better fulfill the therapeutic needs of migraineurs, especially patients suffering from severe and frequent migraine.

There is a growing interest in neuromodulation as a treatment for primary headache disorders. Applying electrical stimulation to relieve pain syndromes is no new approach, but within recent years devices and stimulation protocols have become more accurate and less invasive. While neuromodulation was previously limited to drug-refractory patients, it is now increasingly applied as an alternative to acute and prophylactic therapy (2), raising the question whether it can serve as a valid option in the treatment of migraine.

In addition to the diagnostic criteria specified by the International Headache Society (1), migraine can be associated with cranial autonomic symptoms (CAS) of lacrimation, conjunctival injection, eyelid edema, nasal congestion, and forehead/facial sweating $(3,4)$ in $27 \%$ to $73 \%$ of cases depending on criteria

\footnotetext{
'Danish Headache Center and Department of Neurology, Glostrup Hospital, Faculty of Health \& Medical Sciences, University of Copenhagen, Denmark

${ }^{2}$ Headache Research Unit, University of Liége, Department of Neurology, Citadelle Hospital, Belgium

\section{Corresponding author:}

Messoud Ashina, Danish Headache Center and Department of Neurology, Glostrup Hospital, Faculty of Health \& Medical Sciences, University of Copenhagen, 2600 Glostrup, Denmark.

Email: ashina@dadlnet.dk
} 
and study design (3-6). This correlation is not limited to adults but also includes migraineurs under the age of 18 years, with a recent study showing $62 \%$ of children and adolescents complaining of migraine-associated CAS (7). The presence of CAS in migraineurs suggests activation of the trigemino-autonomic reflex resulting in increased parasympathetic outflow (8), as in the trigeminal autonomic cephalalgias (including cluster headache $(\mathrm{CH})$, chronic paroxysmal hemicranias and short-lasting unilateral neuralgiform headache attacks with conjunctival injection and tearing (SUNCT)). In the latter headache group, clinicians have long attempted to treat attacks and associated symptoms by targeting the sphenopalatine ganglion (SPG), a procedure first introduced at the beginning of the 20th century by Sluder to treat so-called Sluder's neuralgia, a type of trigeminal autonomic cephalalgia (9). Besides pharmacological blocks, SPG interventions have since included surgical or radiofrequency ablations, radiosurgical targeting or lesions (10-13). As the SPG is the major source of parasympathetic innervation not only to the face but also to the cranial cavity, SPG activation could also be a likely contributor to migraine pathophysiology.

A multicenter study (Pathway M1: Sphenopalatine Ganglion Stimulation for the Treatment of Chronic or High Frequency, High-Disability Migraine Headache) is currently under way, a randomized, controlled, interventional, prospective study to evaluate the use of an implanted SPG neurostimulator for the treatment of migraine headache pain, migraine headache symptoms and migraine frequency in high-disability migraineurs (clinicaltrial.gov: NCT01540799). Inclusion criteria comprise subjects reporting at least $75 \%$ of migraine attacks to be fixed unilateral, preferably with associated CAS.

This review will focus on the SPG as a possible target for treating migraine. We will describe the pathophysiology of migraine, concentrating on a parasympathetic dysfunction and the possible role of a hypothalamoparasympathetico-trigeminal triangle. In addition, we will discuss the possible rationale for potential therapeutic effects of SPG neurostimulation in migraine.

\section{Methods}

\section{Search strategy and selection criteria}

We searched PubMed for publications on SPG's influence on migraine, ultimately selecting articles from the period 1908-2013. The keywords were "sphenopalatine ganglion," "migraine," "trigeminovascular pathway," "trigeminal-parasympathetic reflex," "neuromodulation," and "neurostimulation." The search was limited to English language publications and resulting articles were reviewed based on their abstracts to include only pertinent publications, dealing specifically with CAS in migraineurs, anatomical and functional connections between the SPG, cerebral vessels, trigeminovascular system and hypothalamus, and possible therapeutic prospects of SPG neurostimulation.

\section{Anatomo-functional characteristics of the SPG and the trigemino- parasympathetic reflex}

The SPG is the largest extracranial parasympathetic ganglion (Figure 1), with sensory and sympathetic fibers also projecting through the ganglion, however, without synapsing. The parasympathetic fibers originate in the superior salivatory nucleus (SSN) of the pons and supply extra- and intracerebral arteries (14-16), the lacrimal gland $(17,18)$, nasal mucosa $(19,20)$, conjunctiva (20), and Müller's muscle in the upper eyelid (21). Sympathetic fibers originate in the internal carotid plexus to innervate the lacrimal gland, nasal, and palatine mucosa (22). The sensory root originates in the maxillary nerve, supplying the nose, palate, tonsil, and gingiva (22).

Physiologically, low-frequency electrical stimulation of the SPG $(10-20 \mathrm{~Hz})$ in animal studies has revealed dilation of intra- and extracranial arteries, increased cerebral blood flow (CBF), and plasma protein extravasation (PPE) in the dura mater (Table 1). PPE is mediated by sensory neurons that contain potent vasodilator neurotransmitters such as calcitonin generelated peptide (CGRP), substance P (SP), and neurokinin A (NA) (23). As PPE may activate meningeal nociceptors to induce the headache of migraine via neurogenic inflammation $(23,24)$ and dilation of intraand extracranial arteries is related to migraine attacks (25-27), these effects of SPG stimulation contribute to the pathophysiologic understanding of migraine while introducing the prospect of targeting the ganglion with high-frequency stimulation $(100 \mathrm{~Hz})$ for treatment purposes.

The trigemino-parasympathetic reflex consists of a brainstem connection between trigeminal afferents and parasympathetic efferents of the facial nerve that synapse in the SPG $(8,34,35)$. The reflex has been broadly demonstrated in animal and human studies, showing that trigeminal ganglion stimulation leads to intra- and extracranial vasodilation (36-38), increased regional CBF $(39,40)$, and increased facial temperature, a response that is abolished by lesioning the facial nerve (41). Furthermore, painful stimulation of the ophthalmic nerve innervation area results in internal carotid 


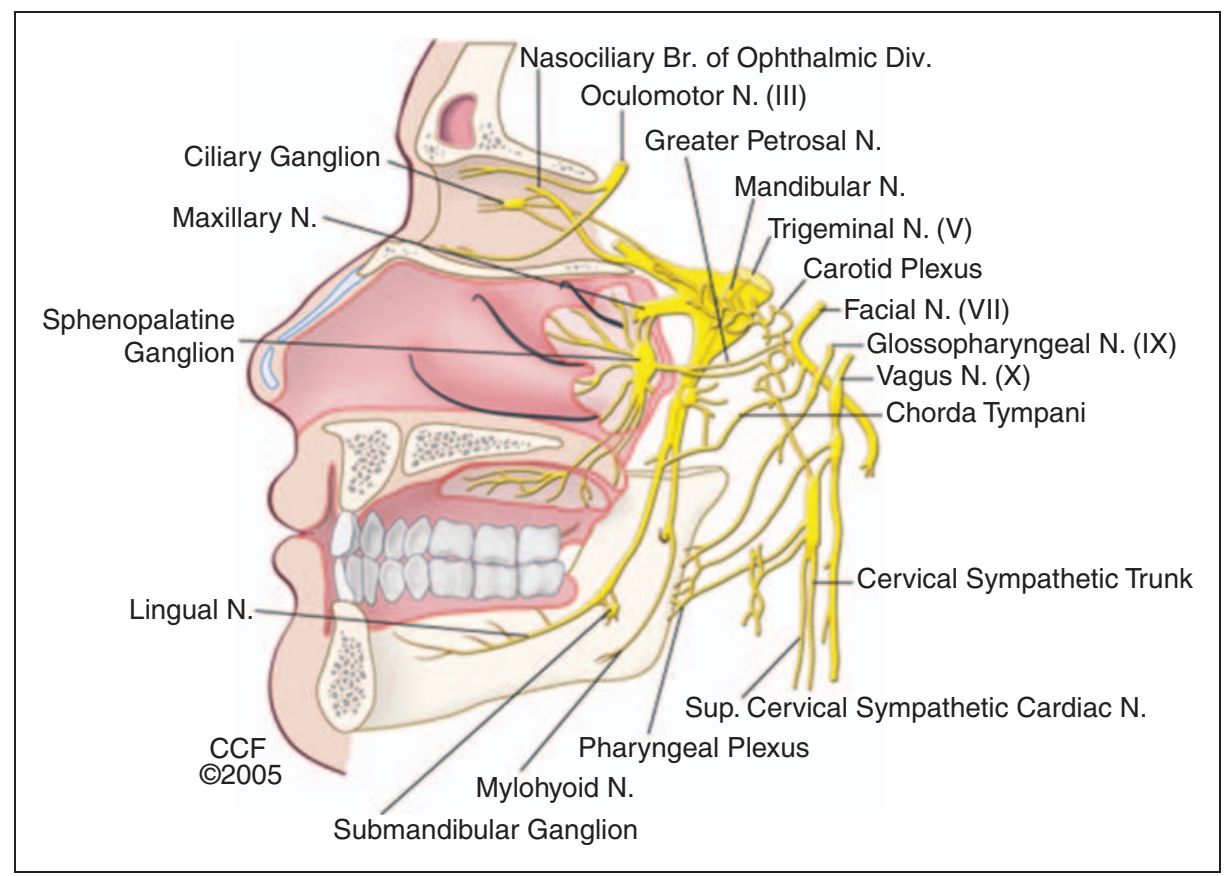

Figure I. Anatomy of the sphenopalatine ganglion (SPG) and associated structures. The SPG is triangular shaped, located in the pterygopalatine fossa, and suspended from the maxillary nerve via the two pterygopalatine nerves. Posteriorly, it is connected to the Vidian nerve formed by the greater petrosal and the deep petrosal nerves. Efferent branches from the ganglion include the superior posterior lateral nasal, nasopalatine, greater and lesser palatine, and pharyngeal nerves. Caudally the ganglion is in direct connection with the greater and lesser palatine nerves.

Source: Reprinted with permission, Cleveland Clinic Center for Medical Art \& Photography (C) 2005-2013. All rights reserved.

artery dilation, CAS, as well as increased ipsilateral blood flow and lacrimal response, of which the latter two effects are also inhibited by facialis lesioning $(38,42-44)$.

\section{Migraine and the parasympathetic nervous system}

Involvement of the autonomic nervous system (ANS) in migraine is considered likely given the symptoms commonly associated with attacks; nausea, emesis, conjunctival injection, lacrimation, nasal congestion, rhinorrhea, salivation, diarrhea, and polyuria (45). Though an alleged autonomic dysfunction has been dealt with widely, migraine pathophysiology is complex, and results have advocated both hypo- and hyperfunction of the sympathetic and parasympathetic systems (Table 2) (46-54).

Involvement of specifically the parasympathetic nervous system is considered plausible as 1) CAS such as lacrimation, rhinorrhea, and eyelid edema are parasympathetic manifestations (55), 2) levels of vasoactive intestinal polypeptide (VIP) are elevated in cranial venous blood during attacks in patients with symptoms of lacrimation and rhinorrhea (56), 3) meningeal blood vessels receive dense parasympathetic innervation, 4) preganglionic parasympathetic neurons in SSN increase their activity after activation of meningeal nociceptors $(57,58)$, and 5) intranasal application of lidocaine, which may block the SPG, is shown to abort migraine within 15 minutes in $36 \%$ of patients in a studied population (11). These latter results should be taken with caution as the effect may be due to blockade of the trigeminovascular afferents passing near the ganglion rather than blockade of the parasympathetic fibers (45). However, Yarnitsky et al. (59) interestingly suggested that SPG might not only play a role in mediating the CAS of migraine but may also contribute to the pain. This is based on their observations that patients with parasympathetic symptoms were more likely to experience pain relief by lidocaine than patients without parasympathetic symptoms, with a pain reduction of $53 \%$ and $15 \%$, respectively.

Avnon et al. (46) studied the trigeminoparasympathetic reflex in migraineurs interictally by instilling soapy eye drops to activate the afferent limb, then measuring subsequent cutaneous vascular and systemic cardiovascular responses as a marker of parasympathetic activity. The authors concluded that autonomic dysfunction in migraine exhibits laterality with parasympathetic hyperfunction in left-sided migraineurs. This concept of asymmetric cerebral function has been suggested previously in studies on the autonomic control of the heart, where the left hemisphere 


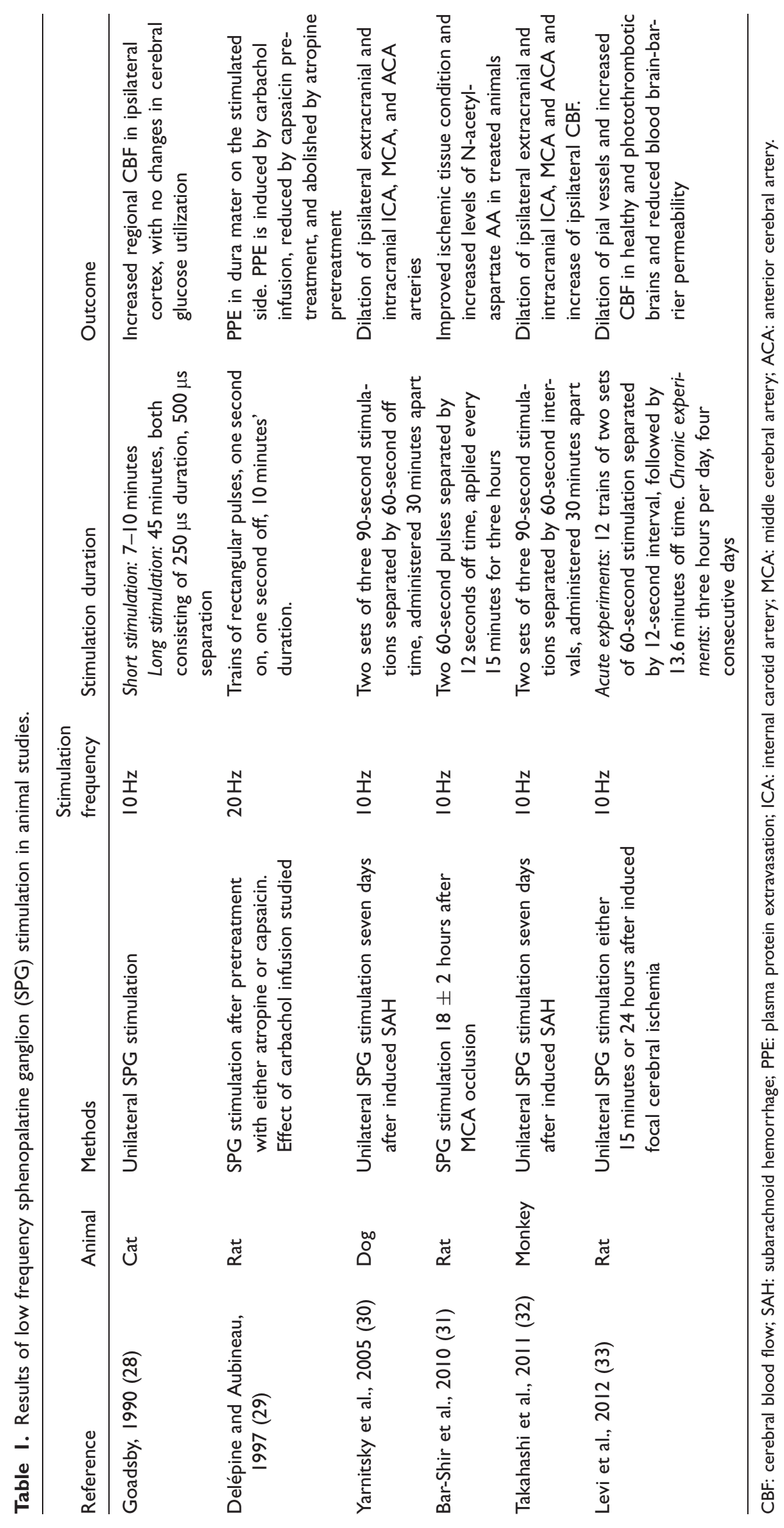


was found to predominantly affect parasympathetic function while the right hemisphere affects sympathetic function (60-63).

Collectively, these data suggest that ANS plays an important role in migraine pathogenesis, where the SPG in particular may mediate parasympathetic hyperactivity considering the efferent fibers, neurotransmitter, and innervation organs of the ganglion (14-16,18-20).

\section{SPG and the migraine pathway: A rationale for aborting migraine with SPG stimulation}

Studies on the effectiveness of SPG stimulation in migraine treatment are still pending. However, assuming a pivotal role of SPG in the pathogenesis and phenotype of migraine based on above considerations, how may we expect stimulation of the ganglion to abort an attack? We propose two hypotheses.

\section{SPG modulates parasympathetic postganglionic outflow}

Burstein and Jakubowski (57) have proposed that common migraine triggers (such as stress and awakening) may activate brain areas that all project to the SSN. The SSN then stimulates the release of parasympathetic neurotransmitters VIP, nitric oxide, and acetyl choline from meningeal terminals of SPG neurons (58). This cascade of parasympathetic activation results in dilation of intracranial blood vessels, PPE, and local release of inflammatory molecules to activate meningeal nociceptors, and thereby induce the migraine headache $(23,57)$. Based on this theory of a common descending pathway that activates meningeal nociceptors, SPG neuromodulation may abort an attack by interrupting the centrally initiated cascade of parasympathetic outflow, possibly by depleting stored neurotransmitter. Inhibiting the activation of the trigeminal afferents may also cancel out triggering of the trigemino-autonomic reflex, thus hindering CAS presentation. Interestingly, Akerman et al. (64) have shown that low-frequency stimulation of the SSN in cats leads to firing of trigeminovascular neurons in the trigeminocervical complex as well as increased blood flow to the lacrimal sac, mediated by increased parasympathetic output. This response emphasizes the prospect that high-frequency SPG stimulation may decrease parasympathetic output, thereby inhibiting the excitatory action on trigeminal nociceptors to alleviate migraine pain and associated autonomic symptoms.

Tepper et al. (65) applied SPG stimulation to treat intractable migraine $(n=11)$, where two patients experienced complete abolition of the headache and 
three experienced pain reduction. While these results may not be conclusive as to the beneficial potential of this treatment, the authors conclude that lack of headache relief appears linked to suboptimal lead placement, poor physiological response, and diagnosis of medication-overuse headache. Ansarinia et al. (66) achieved more promising results in the treatment of acute $\mathrm{CH}$, where complete resolution was obtained in 11 out of 18 attacks, and $>50 \%$ relief in an additional four attacks. Most recently, Schoenen et al. (67) published results of the randomized, sham-controlled pathway $\mathrm{CH}-1$ study of SPG stimulation for chronic $\mathrm{CH}$ $(\mathrm{CCH})$ treatment. This study included in total 28 eligible patients; $67.1 \%$ of attacks were relieved in $64 \%$ of patients and $43 \%$ had a $\geq 50 \%$ reduction in attack frequency. Acute pain relief as well as reduction in attack frequency were reported in $7 \%$ of patients. The authors concluded that SPG stimulation is an effective novel therapy for $\mathrm{CCH}$ sufferers, with a bimodal benefit: acute pain relief and attack prevention. The acute pain relief may arise because of SPG stimulation acting on the trigeminal nociceptive system via an inhibitory information block from the SPG to the target organs of $\mathrm{CH}$ (66). The prophylactic effect, however, may stem from antidromic hypothalamic modulation, the hypothalamus being a main contributor to the pathophysiology of $\mathrm{CH}$ (68-70). Anatomically, hypothalamus modulation through SPG stimulation is feasible as the ganglion receives parasympathetic fibers from the SSN, which in turn receives input from the hypothalamus.

\section{SPG modulates sensory processing in trigeminal nucleus caudalis (TNC)}

Could SPG stimulation result in pain relief due to modulation of sensory processing in the TNC rather than blockade of SPG parasympathetic output? While SPG stimulation has shown to produce mild residual facial pain in the maxillary distribution area (66), migraine headache is experienced in the innervation area of the ophthalmic nerve. Therefore, any pain relief due to sensory processing would presumably stem from a central modulation based on the convergence of trigeminal afferents in the TNC (71), an antinociceptive mechanism similar to that of ONS (72), as occipital nerve fibers also converge centrally with afferents from the first trigeminal branch.

In the parallel discussion of how occipital nerve stimulation (ONS) may be effective in treating $\mathrm{CH}$ patients, Magis et al. $(69,73)$ suggest that ONS works by inducing slow neuromodulatory changes in central pain processing structures, rather than by sensory processing in the TNC. A positron-emission tomography (PET) study performed in $\mathrm{CH}$ patients treated with
ONS showed that hypermetabolism in the painassociated areas (e.g. cingulate gyrus and midbrain) prior to treatment normalized over three to six months with ONS, while hypermetabolism in the hypothalamus remained unchanged (70). These results suggest that ONS acts symptomatically rather than causally, as the study did not prove ONS to alter the activity of the hypothalamus. If we assume that the same mode of action applies to migraineurs, SPG stimulation might act symptomatically because of neuromodulatory changes in central pain processing structures, while not affecting the actual pathophysiology of migraine.

Assuming that our hypothesis of migraine pain alleviation through sensory modulation in TNC is viable, we cannot entirely exclude the consideration that trigeminal neurostimulation in itself may be equally effective, thus not necessarily implicating the SPG. In support of this, a recent randomized controlled trial shows that supraorbital transcutaneous stimulation is effective in preventing migraine (74). Finally, because of the close spatial connection between the SPG and surrounding structures, focal stimulation of the SPG may prove challenging. Therefore, parasympathetic and sensory fibers in the ganglion are possibly targeted simultaneously during SPG stimulation, and our proposed hypotheses of parasympathetic respective sensory modulation may not be mutually exclusive, but rather additive.

\section{Methodological limitations of existing studies on autonomic dysfunction in migraine}

There is currently no scientific consensus on the degree and character of autonomic dysfunction in migraineurs. The results of applied studies are largely contradictory (Table 2), even when comparing studies employing identical tests, indicating that objective verification of ANS dysfunction is complex.

Studies have largely investigated patients exhibiting the large spectrum of autonomic features (including nausea, vomiting, diarrhea, etc) rather than CAS specifically, leading to the question whether these tests have actually investigated the factor relevant to our focus, i.e. cranial autonomic dysfunction as migraine pain and CAS precipitator? Only two studies employ tests that specifically examine the cranial autonomic function, i.e. the trigemino-parasympathetic reflex and pupillometry test. Cardiovascular tests are more widely applied, but is cardiovascular dysfunction necessarily indicative of overall autonomic dysfunction, or indeed cranial dysfunction?

We need a refined test battery to specifically investigate cranial autonomic function in migraineurs to fully assess the possible role of SPG stimulation in migraine treatment. Also, more optimal patient 
stratification is warranted to provide detailed information on the degree of autonomic dysfunction and to increase the specificity of applied tests.

\section{Future perspective}

Based on anatomic and physiological considerations, parasympathetic nervous system involvement in migraine pathophysiology is probable. Therefore, interrupting the autonomic pathway that possibly both generates the pain of migraine and mediates CAS via the trigemino-parasympathetic reflex may serve to inhibit the initiation and presentation of this debilitating primary headache.

We suggest a possible role for SPG stimulation in the treatment of migraine, considering the central role of SPG in mediating parasympathetic outflow to structures of the cranium involved in migraine with CAS, particularly the cranial vessels, meninges, lacrimal gland, nasal mucosa, and conjunctiva. We hypothesize that SPG stimulation may work by either of two mechanisms to terminate migraine; 1 ) interrupting parasympathetic outflow of the ganglion by interfering with preganglionic SSN to SPG efferents or postganglionic outflow, or 2) modulating the sensory processing in TNC, perhaps by way of slow neuromodulatory changes to the pain processing structures of the brain stem. Admittedly, since attack-associated autonomic symptoms are more dramatic in the trigeminal autonomic cephalalgias than in migraine and because outcome in the proof-of-concept study of SPG stimulation during migraine attacks by Tepper et al. (65) was less favorable than that observed with the same method in $\mathrm{CH}$ attacks, one might expect migraineurs to benefit less from SPG stimulation than $\mathrm{CH}$ patients. However, we should be cautious in drawing any firm conclusions based on studies with small sample sizes. Also, there may be a tendency to overlook autonomic symptoms in migraineurs as they are often not spontaneously reported by the patients.
CAS presentation is not a prerequisite for SPG stimulation, as we anticipate analgesia attributed to the effects of neuromodulation. Such pain relief has been suggested to stem from activation of afferent $A \beta$ fibers and gate control in the spinal cord (75) or from a descending supraspinal control from the periaqueductal gray matter and rostroventromedial medulla (76).

Additional clinical trials are needed to fully uncover whether optimal treatment is achieved by stimulating uni- or bilaterally. Prominent symptom and pain laterality may indicate unilateral stimulation, while sideshift occurrence $(68,77)$ may advocate for bilateral stimulation.

A possible role of the hypothalamus in migraine onset is supported by the premonitory symptoms experienced prior to attack onset, the nature of typical migraine triggers (including stress regulation, sleep, food intake, and hormonal changes) and the neuronal connection between hypothalamus and $\operatorname{SSN}(57,78,79)$. Moreover, in a recent imaging study hypothalamic activation was demonstrated during the premonitory phase of migraine attacks (80). Could SPG stimulation then work antidromically to modulate hypothalamic output, thereby preventing the autonomic cascade that initiates migraine? At present, there are no data supporting a hypothalamic effect of SPG stimulation. However, this thesis may be worth investigating further, seeing as the application of neuromodulation to affect upstream components is no new therapeutic concept. Namely, vagus nerve stimulation is widely recognized as an effective treatment of intractable epilepsy, approved by the United States Food and Drug Administration (FDA) in 1997 (81-83).

Overall, more refined studies on autonomic dysfunction in migraineurs will aid in assessing the therapeutic relevance of SPG neurostimulation. Also, future randomized clinical trials using SPG stimulation to treat migraine are warranted to conclude on its true promise as treatment modality as well as to uncover how exactly a beneficial effect is exerted.

\section{Clinical implications}

- Migraine symptoms including parasympathetic manifestations of lacrimation, nasal congestion, conjunctival injection, and parasympathetic activity may also contribute to the pain of migraineurs.

- The sphenopalatine ganglion (SPG) is the largest extracranial parasympathetic ganglion of the head, innervating structures involved in migraine with cephalic autonomic symptoms.

- Because of the association between innervation organs of the SPG and the clinical presentation of migraine, we suggest the prospect of treating migraine with SPG neurostimulation.

- Two possible mechanisms of action are: 1) interrupting the post-ganglionic parasympathetic outflow to inhibit the pain and cephalic autonomic symptoms, and 2) modulating the sensory processing in the trigeminal nucleus caudalis. 


\section{Acknowledgment}

The authors would like to thank Dr Stephen W. Pedersen for valuable input to the paper.

\section{Funding}

This research received no specific grant from any funding agency in the public, commercial, or not-for-profit sectors.

\section{Conflicts of interest}

Dr Messoud Ashina is a principal investigator in the ongoing Pathway M-1 trial: Sphenopalatine Ganglion Stimulation for the Treatment of Chronic or High Frequency, High Disability Migraine Headache (ClinicalTrials.gov Identifier: NCT01540799). The remaining authors have nothing to declare.

\section{References}

1. The International Classification of Headache Disorders. 2nd edition. Cephalalgia 2004; 24 (Suppl) 1: 9-160.

2. Magis D, Jensen R and Schoenen J. Neurostimulation therapies for primary headache disorders: Present and future. Curr Opin Neurol 2012; 25: 269-276.

3. Barbanti P, Fabbrini G, Pesare M, et al. Unilateral cranial autonomic symptoms in migraine. Cephalalgia 2002; 22: 256-259.

4. Lai TH, Fuh JL and Wang SJ. Cranial autonomic symptoms in migraine: Characteristics and comparison with cluster headache. J Neurol Neurosurg Psychiatry 2009; 80: 1116-1119.

5. Gupta R and Bhatia MS. A report of cranial autonomic symptoms in migraineurs. Cephalalgia 2007; 27 : 22-28.

6. Obermann M, Yoon MS, Dommes P, et al. Prevalence of trigeminal autonomic symptoms in migraine: A population-based study. Cephalalgia 2007; 27: 504-509.

7. Gelfand AA, Reider AC and Goadsby PJ. Cranial autonomic symptoms in pediatric migraine are the rule, not the exception. Neurology 2013; 81: 431-436.

8. Goadsby PJ and Lipton RB. A review of paroxysmal hemicranias, SUNCT syndrome and other short-lasting headaches with autonomic feature, including new cases. Brain 1997; 120 (Pt 1): 193-209.

9. Sluder G. The role of the sphenopalatine (or Meckel's) ganglion in nasal headaches. N Y State J Med 1908; 27: $8-13$.

10. Lad SP, Lipani JD, Gibbs IC, et al. Cyberknife targeting the pterygopalatine ganglion for the treatment of chronic cluster headaches. Neurosurgery 2007; 60: E580-E581; discussion E1.

11. Maizels $\mathrm{M}$ and Geiger AM. Intranasal lidocaine for migraine: A randomized trial and open-label follow-up. Headache 1999; 39: 543-551.

12. Narouze S, Kapural L, Casanova J, et al. Sphenopalatine ganglion radiofrequency ablation for the management of chronic cluster headache. Headache 2009; 49: 571-577.

13. Meyer JS, Binns PM, Ericsson AD, et al. Sphenopalatine gangionectomy for cluster headache. Arch Otolaryngol 1970; 92: 475-484.
14. Uddman R, Hara H and Edvinsson L. Neuronal pathways to the rat middle meningeal artery revealed by retrograde tracing and immunocytochemistry. J Auton Nerv Syst 1989; 26: 69-75.

15. Walters BB, Gillespie SA and Moskowitz MA. Cerebrovascular projections from the sphenopalatine and otic ganglia to the middle cerebral artery of the cat. Stroke 1986; 17: 488-494.

16. Hara H, Zhang QJ, Kuroyanagi T, et al. Parasympathetic cerebrovascular innervation: An anterograde tracing from the sphenopalatine ganglion in the rat. Neurosurgery 1993; 32: 822-827; discussion 7.

17. van der Werf F, Baljet B, Prins $M$, et al. Innervation of the lacrimal gland in the cynomolgous monkey: A retrograde tracing study. J Anat 1996; 188 (Pt 3): 591-601.

18. Sibony PA, Walcott B, McKeon C, et al. Vasoactive intestinal polypeptide and the innervation of the human lacrimal gland. Arch Ophthalmol 1988; 106: 1085-1088.

19. Lundberg JM, Anggård A, Emson P, et al. Vasoactive intestinal polypeptide and cholinergic mechanisms in cat nasal mucosa: Studies on choline acetyltransferase and release of vasoactive intestinal polypeptide. Proc Natl Acad Sci U S A 1981; 78: 5255-5259.

20. Ten Tusscher MP, Klooster J, Baljet B, et al. Pre- and post-ganglionic nerve fibres of the pterygopalatine ganglion and their allocation to the eyeball of rats. Brain Res 1990; 517: 315-323.

21. van der Werf F, Baljet B, Prins M, et al. Innervation of the superior tarsal (Müller's) muscle in the cynomolgus monkey: A retrograde tracing study. Invest Ophthalmol Vis Sci 1993; 34: 2333-2340.

22. Moore KL and Dalley AF. Clinically oriented anatomy, 5th edn. Baltimore, USA: Lippincott Williams \& Wilkins, 2006.

23. Moskowitz MA and Macfarlane R. Neurovascular and molecular mechanisms in migraine headaches. Cerebrovasc Brain Metab Rev 1993; 5: 159-177.

24. Moskowitz MA. The neurobiology of vascular head pain. Ann Neurol 1984; 16: 157-168.

25. Iversen $\mathrm{HK}$, Nielsen $\mathrm{TH}$, Olesen $\mathrm{J}$, et al. Arterial responses during migraine headache. Lancet 1990; 336: 837-839.

26. Friberg L, Olesen J, Iversen HK, et al. Migraine pain associated with middle cerebral artery dilatation: Reversal by sumatriptan. Lancet 1991; 338: 13-17.

27. Asghar MS, Hansen AE, Amin FM, et al. Evidence for a vascular factor in migraine. Ann Neurol 2011; 69: 635-645.

28. Goadsby PJ. Sphenopalatine ganglion stimulation increases regional cerebral blood flow independent of glucose utilization in the cat. Brain research 1990; 506: 145-148.

29. Delépine L and Aubineau P. Plasma protein extravasation induced in the rat dura mater by stimulation of the parasympathetic sphenopalatine ganglion. Exp Neurol 1997; 147: 389-400.

30. Yarnitsky D, Lorian A, Shalev A, et al. Reversal of cerebral vasospasm by sphenopalatine ganglion stimulation 
in a dog model of subarachnoid hemorrhage. Surg Neurol 2005; 64: 5-11; discussion 11.

31. Bar-Shir A, Shemesh N, Nossin-Manor R, et al. Late stimulation of the sphenopalatine-ganglion in ischemic rats: Improvement in $\mathrm{N}$-acetyl-aspartate levels and diffusion weighted imaging characteristics as seen by MR. J Magn Reson Imaging 2010; 31: 1355-1363.

32. Takahashi M, Zhang ZD and Macdonald RL. Sphenopalatine ganglion stimulation for vasospasm after experimental subarachnoid hemorrhage. J Neurosurg 2011; 114 : 1104-1109.

33. Levi H, Schoknecht K, Prager O, et al. Stimulation of the sphenopalatine ganglion induces reperfusion and bloodbrain barrier protection in the photothrombotic stroke model. PloS One 2012; 7: e39636.

34. May A and Goadsby PJ. The trigeminovascular system in humans: Pathophysiologic implications for primary headache syndromes of the neural influences on the cerebral circulation. J Cereb Blood Flow Metab 1999; 19: $115-127$.

35. Goadsby PJ, Lipton RB and Ferrari MD. Migraine - current understanding and treatment. N Engl J Med 2002; 346: 257-270.

36. Gonzalez G, Onofrio BM and Kerr FW. Vasodilator system for the face. J Neurosurg 1975; 42: 696-703.

37. Goadsby PJ, Lambert GA and Lance JW. The peripheral pathway for extracranial vasodilatation in the cat. J Auton Nerv Syst 1984; 10: 145-155.

38. Drummond PD. The mechanism of facial sweating and cutaneous vascular responses to painful stimulation of the eye. Brain 1992; 115 (Pt 5): 1417-1428.

39. Goadsby PJ and Duckworth JW. Effect of stimulation of trigeminal ganglion on regional cerebral blood flow in cats. Am J Physiol 1987; 253 (2 Pt 2): R270-R274.

40. Lang $\mathrm{R}$ and Zimmer R. Neurogenic control of cerebral blood flow. Exp Neurol 1974; 43: 143-161.

41. Lambert GA, Bogduk N, Goadsby PJ, et al. Decreased carotid arterial resistance in cats in response to trigeminal stimulation. J Neurosurg 1984; 61: 307-315.

42. Frese A, Evers S and May A. Autonomic activation in experimental trigeminal pain. Cephalalgia 2003; 23: 67-68.

43. May A, Büchel C, Turner R, et al. Magnetic resonance angiography in facial and other pain: Neurovascular mechanisms of trigeminal sensation. J Cereb Blood Flow Metab 2001; 21: 1171-1176.

44. Drummond PD. Lacrimation and cutaneous vasodilatation in the face induced by painful stimulation of the nasal ala and upper lip. J Auton Nerv Syst 1995; 51: 109-116.

45. Olesen J, Goadsby $\mathrm{PJ}$, Ramadan $\mathrm{NH}$, et al. The Headaches, 3rd edn. Philadelphia: Lippincott Williams \& Wilkins, 2006.

46. Avnon Y, Nitzan M, Sprecher E, et al. Autonomic asymmetry in migraine: augmented parasympathetic activation in left unilateral migraineurs. Brain 2004; 127: 2099-2108.

47. Drummond PD. Photophobia and autonomic responses to facial pain in migraine. Brain 1997; 120 (Pt 10): 1857-1864.
48. Micieli G, Tassorelli C, Magri M, et al. Vegetative imbalance in migraine. A dynamic TV pupillometric evaluation. Funct Neurol 1989; 4: 105-111.

49. Havanka-Kanniainen $H$, Tolonen $U$ and Myllylä VV. Autonomic dysfunction in adult migraineurs. Headache 1986; 26: 425-430.

50. Pogacnik T, Sega S, Pecnik B, et al. Autonomic function testing in patients with migraine. Headache 1993; 33: 545-550.

51. Yakinci C, Mungen B, Er H, et al. Autonomic nervous system function in childhood migraine. Pediatr Int 1999; 41: 529-53352.

52. Mosek A, Novak V, Opfer-Gehrking TL, et al. Autonomic dysfunction in migraineurs. Headache 1999; 39: 108-117.

53. Sanya EO, Brown CM, von Wilmowsky C, et al. Impairment of parasympathetic baroreflex responses in migraine patients. Acta Neurol Scand 2005; 111: $102-107$.

54. Thomsen LL and Olesen J. The autonomic nervous system and the regulation of arterial tone in migraine. Clin Auton Res 1995; 5: 243-250.

55. Avnon Y, Nitzan M, Sprecher E, et al. Different patterns of parasympathetic activation in uni- and bilateral migraineurs. Brain 2003; 126: 1660-1670.

56. Goadsby PJ, Edvinsson L and Ekman R. Vasoactive peptide release in the extracerebral circulation of humans during migraine headache. Ann Neurol 1990; 28: 183-187.

57. Burstein R and Jakubowski M. Unitary hypothesis for multiple triggers of the pain and strain of migraine. J Comp Neurol 2005; 493: 9-14.

58. Levy D, Strassman AM and Burstein R. A critical view on the role of migraine triggers in the genesis of migraine pain. Headache 2009; 49: 953-957.

59. Yarnitsky D, Goor-Aryeh I, Bajwa ZH, et al. 2003 Wolff Award: Possible parasympathetic contributions to peripheral and central sensitization during migraine. Headache 2003; 43: 704-714.

60. Hilz MJ, Dutsch M, Perrine K, et al. Hemispheric influence on autonomic modulation and baroreflex sensitivity. Ann Neurol 2001; 49: 575-584.

61. Oppenheimer SM, Gelb A, Girvin JP, et al. Cardiovascular effects of human insular cortex stimulation. Neurology 1992; 42: 1727-1732.

62. Yoon BW, Morillo CA, Cechetto DF, et al. Cerebral hemispheric lateralization in cardiac autonomic control. Arch Neurol 1997; 54: 741-744.

63. Zamrini EY, Meador KJ, Loring DW, et al. Unilateral cerebral inactivation produces differential left/right heart rate responses. Neurology 1990; 40: 1408-1411.

64. Akerman S, Holland PR, Lasalandra MP, et al. Oxygen inhibits neuronal activation in the trigeminocervical complex after stimulation of trigeminal autonomic reflex, but not during direct dural activation of trigeminal afferents. Headache 2009; 49: 1131-1143.

65. Tepper SJ, Rezai A, Narouze S, et al. Acute treatment of intractable migraine with sphenopalatine ganglion electrical stimulation. Headache 2009; 49: 983-989. 
66. Ansarinia M, Rezai A, Tepper SJ, et al. Electrical stimulation of sphenopalatine ganglion for acute treatment of cluster headaches. Headache 2010; 50: 1164-1174.

67. Schoenen J, Jensen RH, Lantéri-Minet $M$, et al. Stimulation of the sphenopalatine ganglion (SPG) for cluster headache treatment. Pathway $\mathrm{CH}-1$ : A randomized, sham-controlled study. Cephalalgia 2013; 33: 816-830.

68. Magis D, Gerardy PY, Remacle JM, et al. Sustained effectiveness of occipital nerve stimulation in drugresistant chronic cluster headache. Headache 2011; 51: 1191-1201.

69. Magis D and Schoenen J. Advances and challenges in neurostimulation for headaches. Lancet Neurol 2012; 11: 708-719.

70. Magis D, Bruno MA, Fumal A, et al. Central modulation in cluster headache patients treated with occipital nerve stimulation: An FDG-PET study. BMC Neurol 2011; 11: 25 .

71. Bartsch T and Goadsby PJ. Stimulation of the greater occipital nerve induces increased central excitability of dural afferent input. Brain 2002; 125: 1496-1509.

72. Burns B, Watkins L and Goadsby PJ. Treatment of intractable chronic cluster headache by occipital nerve stimulation in 14 patients. Neurology 2009; 72: 341-345.

73. Magis D, Allena M, Bolla M, et al. Occipital nerve stimulation for drug-resistant chronic cluster headache: A prospective pilot study. Lancet Neurol 2007; 6: 314-321.

74. Schoenen J, Vandersmissen B, Jeangette S, et al. Migraine prevention with a supraorbital transcutaneous stimulator: A randomized controlled trial. Neurology 2013; 80: 697-704.

75. Melzack R and Wall PD. Pain mechanisms: A new theory. Science 1965; 150: 971-979.

76. DeSantana JM, Da Silva LF, De Resende MA, et al. Transcutaneous electrical nerve stimulation at both high and low frequencies activates ventrolateral periaqueductal grey to decrease mechanical hyperalgesia in arthritic rats. Neuroscience 2009; 163: 1233-1241.

77. Burns B, Watkins L and Goadsby PJ. Treatment of medically intractable cluster headache by occipital nerve stimulation: Long-term follow-up of eight patients. Lancet 2007; 369: 1099-1106.

78. Blau JN. Migraine prodromes separated from the aura: Complete migraine. Br Med J 1980; 281: 658-660.

79. Blau JN. Migraine pathogenesis: The neural hypothesis reexamined. J Neurol Neurosurg Psychiatry 1984; 47: 437-442.

80. Maniyar F, Sprenger T, Schankin C, et al. Imaging the premonitory phase of migraine - new insights into generation of the migraine attack. J Headache Pain 2013; 14(Suppl 1): P112.

81. Koo B. EEG changes with vagus nerve stimulation. J Clin Neurophysiol 2001; 18: 434-441.

82. Englot DJ, Chang EF and Auguste KI. Vagus nerve stimulation for epilepsy: A meta-analysis of efficacy and predictors of response. J Neurosurg 2011; 115: 1248-1255.

83. Krahl SE. Vagus nerve stimulation for epilepsy: A review of the peripheral mechanisms. Surg Neurol Int 2012; 3(Suppl 1): S47-S52. 